Annals of Pure and Applied Mathematics

Vol. 14, No. 1, 2017, 1-10

ISSN: 2279-087X (P), 2279-0888(online)

Published on 1 June 2017

Annals of

www.researchmathsci.org

DOI: http://dx.doi.org/10.22457/apam.v14n1al

Pure and Applied

Mathematics

\title{
Some Cycle Related 4-cordial Graphs
}

\section{N.B.Rathod ${ }^{1}$ and K.K.Kanani ${ }^{2}$}

\author{
${ }^{1}$ School of Science, R.K.University \\ Government Engineering College, Bhavnagar, Gujarat, INDIA. \\ E-mail: rathodneha005@gmail.com \\ ${ }^{2}$ Government Engineering College, Rajkot, Gujarat, INDIA. \\ E-mail: kananikkk@yahoo.co.in
}

Received 11 April 2017; accepted 23 April 2017

Abstract. We discuss here, 4-cordial labeling of some cycle related graphs. We prove that middle graph of the cycle and splitting graph of the cycle are 4-cordial. In addition to this we prove that double wheel and flower graph are 4-cordial.

Keywords: Abelian group; 4-cordial labeling; middle graph; splitting graph; double wheel; flower graph.

AMS Mathematics Subject Classification (2010): 05 C78

\section{Introduction}

Throughout this work, by a graph we mean finite, connected, undirected \& simple graph $G=(V(G), E(G))$ of order $|V(G)|$ and size $|E(G)|$.

Definition 1.1. A graph labeling is an assignment of integers to the vertices or edges or both subject to certain condition(s). If the domain of the mapping is the set of vertices (edges) then the labeling is called a vertex labeling (an edge labeling).

A latest survey on various graph labeling problems can be found in Gallian [1].

Definition 1.2. Let $\langle A, *\rangle$ be any Abelian group. A graph $G=(V(G), E(G))$ is said to be $A$-cordial labeling if there is a mapping $f: V(G) \rightarrow A$ which satisfies the following two conditions when the edge $e=u v$ is labeled as $f(u) * f(v)$

(i) $\quad\left|v_{f}(a)-v_{f}(b)\right| \leq 1$; if for all $a, b \in A$

(ii) $\quad\left|e_{f}(a)-e_{f}(b)\right| \leq 1$; if for all $a, b \in A$

where, $v_{f}(a)=$ the number of vertices with label $a$;

$v_{f}(b)=$ the number of vertices with label $b$;

$e_{f}(a)=$ the number of edges with label $a$;

$e_{f}(b)=$ the number of edges with label $b$.

We note that if $A=\left\langle Z_{k},+k\right\rangle$, that is additive group of modulo $k$ then the labeling is known as $k$-cordial labeling. 


\section{N.B.Rathod and K.K.Kanani}

Here, we consider $A=\left\langle Z_{4},+4\right\rangle$, that is additive group of modulo 4 then the labeling is known as 4-cordial labeling.

The concept of $A$-cordial labeling was introduced by Hovey [3] and proved the following results.

- All the connected graphs are 3-cordial.

- All the trees are 3-cordial.

- All the trees are 4-cordial.

- Cycles are $k$-cordial for all odd $k$.

Kanani and Rathod [4] proved the following results.

- All the Wheels $W_{n}$ are 4-cordial.

- All the fans $f_{n}$ are 4 -cordial.

- All the friendship graphs $F_{n}$ are 4-cordial.

- All the Helms $H_{n}$ are 4-cordial.

Rathod and Kanani [5] proved the following results.

- The middle graph $M\left(P_{n}\right)$ of path $P_{n}$ is 4-cordial.

- The total graph $T\left(P_{n}\right)$ of path $P_{n}$ is 4-cordial.

- The splitting graph $S^{\prime}\left(P_{n}\right)$ of path is 4-cordial.

- The square graph $P_{n}^{2}$ of path is 4-cordial.

- The triangular snake $T S_{n}$ is 4-cordial.

Rathod and Kanani [6]derived the following 4-cordial graphs.

- The splitting graph $S^{\prime}\left(K_{l, n}\right)$ of $\operatorname{star} K_{l, n}$ is 4-cordial.

- The triangular book graph $B_{n}$ is 4-cordial.

- The one point union $f_{3}{ }^{n}$ of $n$ copies of a fan $f_{3}$ is 4-cordial.

Rathod and Kanani [7] proved the following results.

- $\quad$ The graph $Z-P_{n}$ is 4-cordial for all $n$.

- The braid graph $B(n)$ is 4-cordial for all $n$.

- The triangular ladder $T L_{n}$ is 4-cordial for all $n$.

- The irregular quadrilateral snake $I Q\left(S_{n}\right)$ is 4-cordial for all $n$.

\section{Some useful definitions of standard graphs}

1. The Middle Graph $\boldsymbol{M}(\boldsymbol{G})$ of $\mathrm{G}$ is the graph whose vertex set is $V(G) U E(G)$ and in which two vertices are adjacent if and only if either they are adjacent edges of $G$ or one is a vertex of $G$ and the other is an edge incident with it.

2. The Splitting Graph $\boldsymbol{S}^{\prime}(\boldsymbol{G})$ of graph $\mathrm{G}$ is obtained by adding to each vertex $v$ a new vertex $v$ ' such that $v$ ' is adjacent to every vertex which is adjacent to $v$ in $G$ in other words $N(v)=N\left(v^{\prime}\right)$.

3. The Double Wheel Graph $\boldsymbol{D} \boldsymbol{W}_{n}$ of size $n$ can be composed of $2 C_{n}+K_{l}$ it consists of two cycles of size $n$ where vertices of two cycles are all connected to a central vertex.

4. The $\boldsymbol{F l o w e r} \boldsymbol{G r a p h} \boldsymbol{F l}_{\boldsymbol{n}}$ is the graph obtained from a helm $H_{n}$ by joining each pendant vertex to the apex of the helm. 


\section{Some Cycle Related 4-cordial Graphs}

For any undefined term in graph theory we rely upon Gross and Yellen [3].

\section{Main results}

Theorem 2.1. The Middle Graph $M\left(C_{n}\right)$ of cycle $C_{n}$ is 4-cordial.

Proof: Let $G=M\left(C_{n}\right)$ be the middle graph of cycle $C_{n}$ and $v_{l}, v_{2}, \ldots, v_{n}$ be the vertices of $C_{n}$. Let $v^{\prime}{ }_{l}, v_{2}{ }_{2}, \ldots, v_{n}{ }_{n}$ be the vertices added corresponding to the edges $e_{1}, e_{2}, \ldots, e_{n}$ respectively to obtain $M\left(C_{n}\right)$. Here, $|V(G)|=2 n$ and $|E(G)|=3 n$.

To define 4-cordial labeling $f: V(G) \rightarrow Z_{4}$ we consider the following cases.

Case 1: $n \equiv 0(\bmod 8)$.

In this case label the vertices $v_{1}, v_{2}, \ldots, v_{n}$ by

$3,1,2,0,1,3,0,2 ; 3,1,2,0,1,3,0,2 ; \ldots, 1,3,0,2$ and the vertices $v_{1}{ }_{1}, v_{2}{ }_{2}, \ldots, v_{n}{ }_{n}$ by $1,0,3,2 ; 1,0,3,2 ; \ldots, 1,0,3,2$ successively.

Case 2: $n \equiv 1(\bmod 8)$.

In this case label the vertices $v_{1}, v_{2,}, \ldots, v_{n}$ by

$3,1,2,0,1,3,0,2 ; 3,1,2,0,1,3,0,2 ; \ldots, 1,3,0,2,3$ and the vertices $v_{1}{ }_{1}, v_{2,}{ }_{2,}, \ldots, v^{\prime}{ }_{n-2}$ by

$1,0,3,2 ; 1,0,3,2 ; \ldots, 1,0,3$ successively and the vertices $v_{n-1}^{\prime}, v^{\prime}{ }_{n}$ by 1,2 .

Case 3: $n \equiv 2(\bmod 8)$.

In this case label the vertices $v_{1}, v_{2}, \ldots, v_{n-1}$ successively by $3,1,2,0,1,3,0,2 ; 3,1,2,0,1,3,0,2 ; \ldots, 1,3,0,2,3$ and the vertex $v_{n}$ by 2 .

Label the vertices $v_{1}{ }_{1}, v_{2}{ }_{2}, \ldots, v_{n}{ }_{n}$ by $1,0,3,2 ; 1,0,3,2 ; \ldots, 1,0,3,2,1,0$ successively.

Case 4: $n \equiv 3(\bmod 8)$.

In this case label the vertices $v_{1}, v_{2}, \ldots, v_{n}$ by

$3,1,2,0,1,3,0,2 ; 3,1,2,0,1,3,0,2 ; \ldots, 1,3,0,2,3,1,2$. Label the vertices $v_{1}{ }_{1}, v_{2}{ }_{2,}, \ldots, v_{n-1}$

successively by $1,0,3,2 ; 1,0,3,2 ; \ldots, 1,0,3,2,1,0$ and the vertex $v_{n}^{\prime}$ by 0 .

Case 5: $n \equiv 4(\bmod 8)$.

In this case label the vertices $v_{1}, v_{2}, \ldots, v_{n-2}$ successively by

$3,1,2,0,1,3,0,2 ; 3,1,2,0,1,3,0,2 ; \ldots, 1,3,0,2,3,1$ and the vertices $v_{n-1}, v_{n}$ by 3,0 .

Label the vertices $v^{\prime}{ }_{1}, v_{2}{ }_{2}, \ldots, v_{n-2}$ successively by $1,0,3,2 ; 1,0,3,2 ; \ldots, 1,0,3,2,1,0$ and the vertices $v_{n-1}^{\prime}, v_{n}^{\prime}$ by 2,2 .

Case 6: $n \equiv 5(\bmod 8)$.

In this case label the vertices $v_{1}, v_{2}, \ldots, v_{n}$ successively by

$3,1,2,0,1,3,0,2 ; 3,1,2,0,1,3,0,2 ; \ldots, 1,3,0,2,3,1,2,0,1$. Label the vertices $v_{1}{ }_{1}, v_{2}{ }_{2,}, \ldots, v_{n-1}$ successively by $1,0,3,2 ; 1,0,3,2 ; \ldots, 1,0,3,2,1,0,3,2$ and the vertex $v^{\prime}{ }_{n}$ by 0 .

Case 7: $n \equiv 6(\bmod 8)$.

In this case label the vertices $v_{1}, v_{2}, \ldots, v_{n-2}$ successively by

$3,1,2,0,1,3,0,2 ; 3,1,2,0,1,3,0,2 ; \ldots, 1,3,0,2,3,1,2,0$ and the vertices $v_{n-1}, v_{n}$ by 3,1 .

Label the vertices $v^{\prime}{ }_{1}, v_{2}{ }_{2,}, \ldots, v^{\prime}{ }_{n-2}$ successively by $1,0,3,2 ; 1,0,3,2 ; \ldots, 1,0,3,2,1,0,3,2$ and the vertices $v_{n-1}^{\prime}, v_{n}^{\prime}$ by 0,2 .

Case 8: $n \equiv 7(\bmod 8)$.

In this case label the vertices $v_{l}, v_{2}, \ldots, v_{n-1}$ successively by 


\section{N.B.Rathod and K.K.Kanani}

$3,1,2,0,1,3,0,2 ; 3,1,2,0,1,3,0,2 ; \ldots, 1,3,0,2,3,1,2,0,1,3$ and the vertex $v_{n}$ by 1 .

Label the vertices $v^{\prime}{ }_{1}, v^{\prime}{ }_{2}, \ldots, v_{n-3}$ successively by $1,0,3,2 ; 1,0,3,2 ; \ldots, 1,0,3,2,1,0,3,2$ and the vertices $v_{n-2}^{\prime}, v_{n-1}^{\prime}, v_{n}^{\prime}$ by $2,0,3$.

Table 1 show that above defined labeling pattern satisfies the vertex conditions and edge conditions for 4-cordial labeling. Hence, the Middle Graph $M\left(C_{n}\right)$ of cycle $C_{n}$ is 4cordial.

Let $n=8 a+b, a, b \in N U\{0\}$.

\begin{tabular}{|c|c|c|}
\hline $\mathrm{b}$ & Vertex conditions & Edge conditions \\
\hline 0,4 & $v_{f}(0)=v_{f}(1)=v_{f}(2)=v_{f}(3)$ & $e_{f}(0)=e_{f}(1)=e_{f}(2)=e_{f}(3)$ \\
\hline 1 & $v_{f}(0)+1=v_{f}(1)=v_{f}(2)+1=v_{f}(3)$ & $e_{f}(0)+1=e_{f}(1)=e_{f}(2)=e_{f}(3)$ \\
\hline 2,6 & $v_{f}(0)=v_{f}(1)=v_{f}(2)=v_{f}(3)$ & $e_{f}(0)+1=e_{f}(1)=e_{f}(2)+1=e_{f}(3)$ \\
\hline 3 & $v_{f}(0)=v_{f}(1)=v_{f}(2)+1=v_{f}(3)+1$ & $e_{f}(0)+1=e_{f}(1)+1=e_{f}(2)=e_{f}(3)+1$ \\
\hline 5 & $v_{f}(0)=v_{f}(1)=v_{f}(2)+1=v_{f}(3)+1$ & $e_{f}(0)+1=e_{f}(1)=e_{f}(2)=e_{f}(3)$ \\
\hline 7 & $v_{f}(0)+1=v_{f}(1)=v_{f}(2)+1=v_{f}(3)$ & $e_{f}(0)=e_{f}(1)+1=e_{f}(2)+1=e_{f}(3)+1$ \\
\hline
\end{tabular}

Table 1:

Illustration 2.2. The Middle graph $M\left(C_{5}\right)$ and its 4-cordial labeling is shown in Figure 1 .

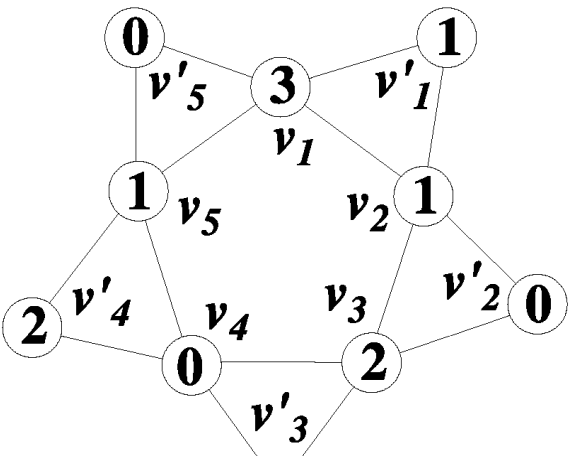

3

Figure 1: 4-cordial labeling of middle graph $\mathrm{M}\left(\mathrm{C}_{5}\right)$ of cycle $\mathrm{C}_{5}$ graph.

Theorem 2.3. The splitting graph $S^{\prime}\left(C_{n}\right)$ of cycle $C_{n}$ is 4-cordial.

Proof: Let $\mathrm{G}=S^{\prime}\left(C_{n}\right)$ be the splitting graph of cycle $C_{n}$ and $v_{1}, v_{2}, \ldots, v_{n}$ be the vertices of $C_{n}$. Let $G$ be the graph obtained by adding to each vertex $v$ a new vertex $v$ ' such that $v$ ' is adjacent to every vertex that is adjacent to $v$ in $G$. Here, $|V(G)|=2 n$ and $|E(G)|=3 n$. To define 4-cordial labeling $f: V(G) \rightarrow Z_{4}$ we consider the following cases.

Case 1: $n \equiv 0(\bmod 8)$.

In this case label the vertices $v_{1}, v_{2}, \ldots, v_{n}$ by 
Some Cycle Related 4-cordial Graphs

$3,1,2,0,1,3,0,2 ; 3,1,2,0,1,3,0,2 ; \ldots, 1,3,0,2$ and the vertices $v^{\prime}{ }_{1}, v^{\prime}{ }_{2,}, \ldots, v_{n}{ }_{n}$ by $3,1,2,0,1,3,0,2 ; 3,1,2,0,1,3,0,2 ; \ldots, 1,3,0,2$ successively.

Case 2: $n \equiv 1(\bmod 8)$.

In this case label the vertices $v_{1}, v_{2}, \ldots, v_{n-1}$ successively by $3,1,2,0,1,3,0,2 ; 3,1,2,0,1,3,0,2 ; \ldots, 1,3,0,2$ and the vertex $v_{n}$ by 2 . Label the vertices $v_{1}{ }_{1}, v_{2}{ }_{2}, \ldots, v_{n-2}$ successively by $3,1,2,0,1,3,0,2 ; 3,1,2,0,1,3,0,2 ; \ldots, 1,3,0$ and the vertices $v_{n-1}^{\prime}, v_{n}^{\prime}$ by 1,3 .

Case 3: $n \equiv 2(\bmod 8)$.

In this case label the vertices $v_{1}, v_{2}, \ldots, v_{n-2}$ successively by $3,1,2,0,1,3,0,2 ; 3,1,2,0,1,3,0,2 ; \ldots, 1,3,0,2$ and the vertices $v_{n-1}, v_{n}$ by 0,2 .

Label the vertices $v^{\prime}{ }_{1}, v_{2,}{ }_{2}, \ldots, v^{\prime}{ }_{n-3}$ successively by $3,1,2,0,1,3,0,2 ; 3,1,2,0,1,3,0,2 ; \ldots, 1,3,0$ and the vertices $v_{n-2}^{\prime}, v_{n-1}^{\prime}, v_{n}^{\prime}$ by $1,2,3$.

Case 4: $n \equiv 3(\bmod 8)$.

In this case label the vertices $v_{1}, v_{2}, \ldots, v_{n}$ successively by $3,1,2,0,1,3,0,2 ; 3,1,2,0,1,3,0,2 ; \ldots, 1,3,0,2,3,1,2$. Label the vertices $v^{\prime}{ }_{1}, v_{2}{ }_{2,}, \ldots, v_{n-2}$ successively by $3,1,2,0,1,3,0,2 ; 3,1,2,0,1,3,0,2 ; \ldots, 1,3,0,2,3$ and the vertices $v_{n-1}^{\prime}, v_{n}{ }_{n}$ by 0,1 .

Case 5: $n \equiv 4(\bmod 8)$.

In this case label the vertices $v_{1}, v_{2}, \ldots, v_{n-1}$ successively by $3,1,2,0,1,3,0,2 ; 3,1,2,0,1,3,0,2 ; \ldots, 1,3,0,2,3,1,2$ and the vertex $v_{n}$ by 3 .

Label the vertices $v_{1}{ }_{1}, v_{2}{ }_{2}, \ldots, v_{n-4}$ successively by $3,1,2,0,1,3,0,2 ; 3,1,2,0,1,3,0,2 ; \ldots, 1,3,0,2$ and the vertices $v_{n-3}^{\prime}, v_{n-2}^{\prime}, v_{n-1}^{\prime}, v_{n}^{\prime}$ by $0,1,0,2$.

Case 6: $n \equiv 5(\bmod 8)$.

In this case label the vertices $v_{1}, v_{2,}, \ldots, v_{n-1}$ successively by $3,1,2,0,1,3,0,2 ; 3,1,2,0,1,3,0,2 ; \ldots, 1,3,0,2,3,1,2,0$ and the vertex $v_{n}$ by 2 . Label the vertices $v^{\prime}{ }_{1}, v_{2,}{ }_{2, \ldots}, v^{\prime}{ }_{n-2}$ successively by $3,1,2,0,1,3,0,2 ; 3,1,2,0,1,3,0,2 ; \ldots, 1,3,0,2,3,1,2$ and the vertices $v_{n-1}^{\prime}, v_{n}^{\prime}$ by 3,0 .

Case 7: $n \equiv 6(\bmod 8)$.

In this case label the vertices $v_{1}, v_{2}, \ldots, v_{n-1}$ successively by $3,1,2,0,1,3,0,2 ; 3,1,2,0,1,3,0,2 ; \ldots, 1,3,0,2,3,1,2,0,1$ and the vertex $v_{n}$ by 2 .

Label the vertices $v^{\prime}{ }_{1}, v_{2}{ }_{2}, \ldots, v^{\prime}{ }_{n-2}$ successively by $3,1,2,0,1,3,0,2 ; 3,1,2,0,1,3,0,2 ; \ldots, 1,3,0,2,3,1,2,0$ and the vertices $v_{n-1}^{\prime}, v_{n}^{\prime}$ by 0,3 .

Case 8: $n \equiv 7(\bmod 8)$.

In this case label the vertices $v_{1}, v_{2}, \ldots, v_{n-1}$ successively by $3,1,2,0,1,3,0,2 ; 3,1,2,0,1,3,0,2 ; \ldots, 1,3,0,2,3,1,2,0,1,3$ and the vertex $v_{n}$ by 2 .

Label the vertices $v_{1}{ }_{1}, v_{2}{ }_{2}, \ldots, v_{n-3}{ }_{n-3}$ successively by $3,1,2,0,1,3,0,2 ; 3,1,2,0,1,3,0,2 ; \ldots, 1,3,0,2,3,1,2,0$ and the vertices $v_{n-2}^{\prime}, v_{n-1}^{\prime}, v_{n}^{\prime}$ by $0,1,3$.

Table 2 show that above defined labeling pattern satisfies the vertex conditions and edge conditions for 4-cordial labeling. Hence, the Splitting Graph $S^{\prime}\left(C_{n}\right)$ of cycle $C_{n}$ is 4cordial. 
Let $n=8 a+b, a, b \in N U\{0\}$.

\begin{tabular}{|c|c|c|}
\hline $\mathrm{b}$ & Vertex conditions & Edge conditions \\
\hline 0,4 & $v_{f}(0)=v_{f}(1)=v_{f}(2)=v_{f}(3)$ & $e_{f}(0)=e_{f}(1)=e_{f}(2)=e_{f}(3)$ \\
\hline 1 & $v_{f}(0)+1=v_{f}(1)=v_{f}(2)+1=v_{f}(3)$ & $e_{f}(0)=e_{f}(1)=e_{f}(2)+1=e_{f}(3)$ \\
\hline 2 & $v_{f}(0)=v_{f}(1)=v_{f}(2)=v_{f}(3)$ & $e_{f}(0)=e_{f}(1)+1=e_{f}(2)=e_{f}(3)+1$ \\
\hline 3 & $v_{f}(0)=v_{f}(1)+1=v_{f}(2)+1=v_{f}(3)+1$ & $e_{f}(0)+1=e_{f}(1)=e_{f}(2)+1=e_{f}(3)$ \\
\hline 5 & $v_{f}(0)+1=v_{f}(1)+1=v_{f}(2)=v_{f}(3)$ & $e_{f}(0)=e_{f}(1)=e_{f}(2)+1=e_{f}(3)$ \\
\hline 6 & $v_{f}(0)+1=v_{f}(1)=v_{f}(2)=v_{f}(3)+1$ & $e_{f}(0)=e_{f}(1)+1=e_{f}(2)=e_{f}(3)+1$ \\
\hline 7 & $v_{f}(0)+1=v_{f}(1)=v_{f}(2)+1=v_{f}(3)$ & $e_{f}(0)+1=e_{f}(1)+1=e_{f}(2)=e_{f}(3)+1$ \\
\hline
\end{tabular}

\section{Table 2:}

Illustration 2.4. The Splitting graph $S^{\prime}\left(C_{8}\right)$ and its 4-cordial labeling is shown in Figure 2.

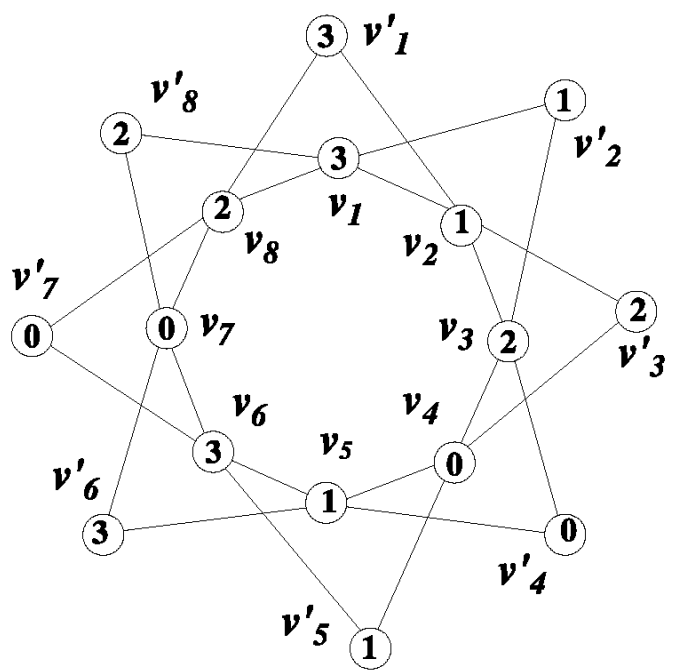

Figure 2: 4-cordial labeling of splitting graph $\mathrm{S}^{\prime}\left(\mathrm{C}_{8}\right)$ of cycle $\mathrm{C}_{8}$ graph.

Theorem 2.5. The double wheel graph $D W_{n}$ is 4-cordial.

Proof: Let $\mathrm{G}=D W_{n}$ be the double wheel graph. Let $v_{l}, v_{2}, \ldots, v_{n}$ be the $n$ vertices of first wheel and $v^{\prime}{ }_{1}, v^{\prime}, \ldots, v_{n}{ }_{n}$ be the vertices of other wheel. Let $v$ be the apex vertex, which is common for both the wheels. Here, $|V(G)|=2 n+1$ and $|E(G)|=4 n$.

To define 4-cordial labeling $f: V(G) \rightarrow Z_{4}$ we consider the following cases.

Label the apex vertex $v$ by 0 .

Case 1: $n \equiv 0(\bmod 8)$.

In this case label the vertices $v_{1}, v_{2}, \ldots, v_{n}$ by

$1,3,2,0,3,1,2,0 ; 1,3,2,0,3,1,2,0 ; \ldots, 3,1,2,0$ and the vertices $v^{\prime}{ }_{1}, v_{2,}{ }_{2,}, \ldots, v^{\prime}{ }_{n}$ by

$1,3,0,2,3,1,0,2 ; 1,3,0,2,3,1,0,2 ; \ldots, 3,1,0,2$ successively. 


\section{Some Cycle Related 4-cordial Graphs}

Case 2: $n \equiv 1(\bmod 8)$.

In this case label the vertices $v_{1}, v_{2}, \ldots, v_{n}$ successively by $1,3,2,0,3,1,2,0 ; 1,3,2,0,3,1,2,0 ; \ldots, 3,1,2,0,1$. Label the vertices $v_{1}{ }_{1}, v_{2,}, \ldots, v_{n-3}{ }_{n-3}$ successively by $1,3,0,2,3,1,0,2 ; 1,3,0,2,3,1,0,2 ; \ldots, 3,1$ and the vertices $v_{n-2}^{\prime}, v_{n-1}^{\prime}, v_{n}^{\prime}$ by $2,2,3$.

Case 3: $n \equiv 2(\bmod 8)$.

In this case label the vertices $v_{1}, v_{2}, \ldots, v_{n-5}$ successively by

$1,3,2,0,3,1,2,0 ; 1,3,2,0,3,1,2,0 ;, \ldots, 3$ and the vertices $v_{n-4}, v_{n-3}, v_{n-2}, v_{n-1}, v_{n}$ by 2,2,1,2,0.

Label the vertices $v^{\prime}{ }_{1}, v^{\prime}{ }_{2}, \ldots, v^{\prime}{ }_{n}$ successively by $1,3,0,2,3,1,0,2 ; 1,3,0,2,3,1,0,2 ; \ldots, 3,1$

,0,2,1,3.

Case 4: $n \equiv 3(\bmod 8)$.

In this case label the vertices $v_{1}, v_{2}, \ldots, v_{n-1}$ successively by

$1,3,2,0,3,1,2,0 ; 1,3,2,0,3,1,2,0 ;, \ldots, 3,1,2,0,1,3$ and the vertex $v_{n}$ by 3 . Label the vertices $v^{\prime}{ }_{1}, v^{\prime}{ }_{2}, \ldots, v_{n-2}$ successively by $1,3,0,2,3,1,0,2 ; 1,3,0,2,3,1,0,2 ; \ldots, 3,1,0,2,1$ and the vertices

$v_{n-1}^{\prime}, v_{n}^{\prime}$ by 2,0 .

Case 5: $n \equiv 4(\bmod 8)$.

In this case label the vertices $v_{1}, v_{2,}, \ldots, v_{n}$ successively by $1,3,2,0,3,1,2,0 ; 1,3,2,0,3,1,2,0 ;, \ldots, 3,1,2,0,1,3,2,0$ and the vertices $v^{\prime}{ }_{1}, v^{\prime}{ }_{2}, \ldots, v^{\prime}{ }_{n}$ successively by $1,3,0,2,3,1,0,2 ; 1,3,0,2,3,1,0,2 ; \ldots, 3,1,0,2,1,3,0,2$.

Case 6: $n \equiv 5(\bmod 8)$.

In this case label the vertices $v_{1}, v_{2}, \ldots, v_{n}$ successively by

$1,3,2,0,3,1,2,0 ; 1,3,2,0,3,1,2,0 ;, \ldots, 3,1,2,0,1,3,2,0,3$. Label the vertices $v_{1}{ }_{1}, v_{2,}, \ldots, v_{n-3}$ successively by $1,3,0,2,3,1,0,2 ; 1,3,0,2,3,1,0,2 ; \ldots, 3,1,0,2,1,3$ and the vertices $v_{n-2}^{\prime}, v_{n-1}^{\prime}$ , $v_{n}$ by $2,2,1$.

Case 7: $n \equiv 6(\bmod 8)$.

In this case label the vertices $v_{1}, v_{2}, \ldots, v_{n-2}$ successively by $1,3,2,0,3,1,2,0 ; 1,3,2,0,3,1,2,0 ;, \ldots, 3,1,2,0,1,3,2,0$ and the vertices $v_{n-1}, v_{n}$ by 1,2 .

Label the vertices $v^{\prime}{ }_{1}, v_{2}{ }_{2}, \ldots, v^{\prime}{ }_{n-2}$ successively by $1,3,0,2,3,1,0,2 ; 1,3,0,2,3,1,0,2 ; \ldots, 3,1,0,2,1,3,0,2$ and the vertices $v_{n-1}^{\prime}, v^{\prime}{ }_{n}$ by 2,3 .

Case 8: $n \equiv 7(\bmod 8)$.

In this case label the vertices $v_{1}, v_{2}, \ldots, v_{n-3}$ successively by $1,3,2,0,3,1,2,0 ; 1,3,2,0,3,1,2,0 ;, \ldots, 3,1,2,0,1,3,2,0$ and the vertices $v_{n-2}, v_{n-1} . v_{n}$ by $2,1,3$. Label the vertices $v_{1}{ }_{1}, v_{2}, \ldots, v_{n-2}$ successively by $1,3,0,2,3,1,0,2 ; 1,3,0,2,3,1,0,2 ; \ldots, 3,1,0,2,1,3,0,2,3$ and the vertices $v_{n-1}^{\prime}, v_{n}^{\prime}$ by 0,1 .

Table 3 show that above defined labeling pattern satisfies the vertex conditions and edge conditions for 4-cordial labeling. Hence, the Double wheel Graph $D W_{n}$ is 4-cordial. 
Let $n=8 a+b, a, b \in N U\{0\}$.

\begin{tabular}{|c|l|c|}
\hline $\mathrm{b}$ & \multicolumn{1}{|c|}{ Vertex conditions } & Edge conditions \\
\hline 0,4 & $v_{f}(0)=v_{f}(1)+1=v_{f}(2)+1=v_{f}(3)+1$ & $e_{f}(0)=e_{f}(1)=e_{f}(2)=e_{f}(3)$ \\
\hline 1,5 & $v_{f}(0)+1=v_{f}(1)=v_{f}(2)=v_{f}(3)$ & $e_{f}(0)=e_{f}(1)=e_{f}(2)=e_{f}(3)$ \\
\hline 2,6 & $v_{f}(0)+1=v_{f}(1)+1=v_{f}(2)=v_{f}(3)+1$ & $e_{f}(0)=e_{f}(1)=e_{f}(2)=e_{f}(3)$ \\
\hline 3,7 & $v_{f}(0)=v_{f}(1)=v_{f}(2)+1=v_{f}(3)$ & $e_{f}(0)=e_{f}(1)=e_{f}(2)=e_{f}(3)$ \\
\hline
\end{tabular}

Table 3:

Illustration 2.6. The double wheel graph $D W_{6}$ and its 4-cordial labeling is shown in Figure 3.

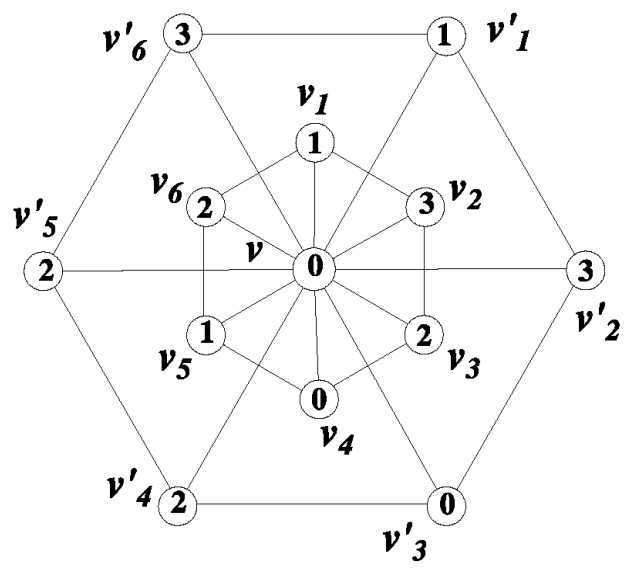

Figure 3: 4-cordial labeling of double wheel graph $\mathrm{DW}_{6}$.

Theorem 2.7. The flower graph $F L_{n}$ is 4-cordial.

Proof: Let $\mathrm{G}=F L_{n}$ be the Flower graph obtained from the helm $H_{n}$. Let $v$ be the apex vertex and $v_{1}, v_{2}, \ldots, v_{n}$ be the $n$ vertices of cycle and $v_{1}^{\prime}, v_{2}{ }_{2}, \ldots, v_{n}{ }_{n}$ be the pendant vertices. The flower graph is obtained from helm $H_{n}$ by joining each pendant vertex to the apex vertex of helm. Here, $|V(G)|=2 n+1$ and $|E(G)|=4 n$.

To define 4-cordial labeling $f: V(G) \rightarrow Z_{4}$ we consider the following cases.

Label the apex vertex $v$ by 0 .

Case 1: $n \equiv 0(\bmod 8)$.

In this case label the vertices $v_{1}, v_{2}, \ldots, v_{n}$ by

$1,3,2,0,3,1,2,0 ; 1,3,2,0,3,1,2,0 ; \ldots, 3,1,2,0$ and the vertices $v_{1}{ }_{1}, v_{2}{ }_{2,}, \ldots, v_{n}{ }_{n}$ by

$1,0,3,2,1,0,2,3 ; 1,0,3,2,1,0,2,3 ; \ldots, 1,0,2,3$ successively.

Case 2: $n \equiv 1(\bmod 8)$.

In this case label the vertices $v_{1}, v_{2}, \ldots, v_{n}$ successively by

$1,3,2,0,3,1,2,0 ; 1,3,2,0,3,1,2,0 ; \ldots, 3,1,2,0,1$. Label the vertices $v_{1}{ }_{1}, v_{2,}, \ldots, v_{n-1}$ successively by $1,0,3,2,1,0,2,3 ; 1,0,3,2,1,0,2,3 ; \ldots, 1,0,2,3$ and the vertex $v^{\prime}{ }_{n}$ by 3 . 
Case 3: $n \equiv 2(\bmod 8)$.

In this case label the vertices $v_{1}, v_{2}, \ldots, v_{n-2}$ successively by $1,3,2,0,3,1,2,0 ; 1,3,2,0,3,1,2,0 ;, \ldots, 3,1,2,0$ and the vertices $v_{n-1}, v_{n}$ by 3,1 .

Label the vertices $v_{1}{ }_{1}, v_{2,}{ }_{2,} \ldots, v_{n-2}$ successively by $1,0,3,2,1,0,2,3 ; 1,0,3,2,1,0,2,3 ; \ldots, 1,0,2,3$ and the vertices $v_{n-1}^{\prime}, v_{n}{ }_{n}$ by 2,0 .

Case 4: $n \equiv 3(\bmod 8)$.

In this case label the vertices $v_{1}, v_{2}, \ldots, v_{n}$ successively by $1,3,2,0,3,1,2,0 ; 1,3,2,0,3,1,2,0 ;, \ldots, 3,1,2,0,1,3,2$. Label the vertices $v_{1}{ }_{1}, v_{2}{ }_{2}, \ldots, v_{n-1}{ }_{n-1}$ successively by $1,0,3,2,1,0,2,3 ; 1,0,3,2,1,0,2,3 ; \ldots, 1,0,2,3,1,0$ and the vertex $v^{\prime}{ }_{n}$ by 2 .

Case 5: $n \equiv 4(\bmod 8)$.

In this case label the vertices $v_{1}, v_{2}, \ldots, v_{n-1}$ successively by $1,3,2,0,3,1,2,0 ; 1,3,2,0,3,1,2,0 ;, \ldots, 3,1,2,0,1,3,2$ and the vertex $v_{n}$ by 2 . Label the vertices $v^{\prime}{ }_{1}, v_{2,}{ }_{2}, \ldots, v_{n}{ }_{n}$ successively by $1,0,3,2,1,0,2,3 ; 1,0,3,2,1,0,2,3 ; \ldots, 1,0,2,3,1,0,3,2$.

Case 6: $n \equiv 5(\bmod 8)$.

In this case label the vertices $v_{1}, v_{2}, \ldots, v_{n}$ successively by $1,3,2,0,3,1,2,0 ; 1,3,2,0,3,1,2,0 ;, \ldots, 3,1,2,0,1,3,2,0,3$. Label the vertices $v_{1}{ }_{1}, v_{2}{ }_{2,}, \ldots, v_{n}{ }_{n}$ successively by $1,0,3,2,1,0,2,3 ; 1,0,3,2,1,0,2,3 ; \ldots, 1,0,2,3,1,0,3,2,1$.

Case 7: $n \equiv 6(\bmod 8)$.

In this case label the vertices $v_{1}, v_{2}, \ldots, v_{n}$ successively by $1,3,2,0,3,1,2,0 ; 1,3,2,0,3,1,2,0 ;, \ldots, 3,1,2,0,1,3,2,0,3,1$.

Label the vertices $v_{1}{ }_{1}, v_{2,}{ }_{2, \ldots,} v_{n-2}$ successively by $1,0,3,2,1,0,2,3 ; 1,0,3,2,1,0,2,3 ; \ldots, 1,0,3,2,1,0,2,3$ and the vertices $v_{n-1}^{\prime}, v_{n}^{\prime}$ by 2,0 .

Case 8: $n \equiv 7(\bmod 8)$.

In this case label the vertices $v_{1}, v_{2}, \ldots, v_{n-3}$ successively by $1,3,2,0,3,1,2,0 ; 1,3,2,0,3,1,2,0 ;, \ldots, 3,1,2,0,1,3,2,0$ and the vertices $v_{n-2}, v_{n-1} . v_{n}$ by $2,1,3$. Label the vertices $v_{1}{ }_{1}, v_{2,}{ }_{2, \ldots,} v_{n-1}{ }_{n-1}$ successively by $1,0,3,2,1,0,2,3 ; 1,0,3,2,1,0,2,3 ; \ldots, 1,0,3,2,1,0,2,3,1,0$ and the vertex $v^{\prime}{ }_{n}$ by 2 .

Table 4 show that above defined labeling pattern satisfies the vertex conditions and edge conditions for 4-cordial labeling. Hence, the Flower Graph $F L_{n}$ is 4-cordial.

Let $n=8 a+b, a, b \in N U\{0\}$.

\begin{tabular}{|c|l|c|}
\hline $\mathrm{b}$ & \multicolumn{1}{|c|}{ Vertex conditions } & Edge conditions \\
\hline $0,2,6$ & $v_{f}(0)=v_{f}(1)+1=v_{f}(2)+1=v_{f}(3)+1$ & $e_{f}(0)=e_{f}(1)=e_{f}(2)=e_{f}(3)$ \\
\hline 1,5 & $v_{f}(0)=v_{f}(1)=v_{f}(2)+1=v_{f}(3)$ & $e_{f}(0)=e_{f}(1)=e_{f}(2)=e_{f}(3)$ \\
\hline 4 & $v_{f}(0)+1=v_{f}(1)+1=v_{f}(2)=v_{f}(3)+1$ & $e_{f}(0)=e_{f}(1)=e_{f}(2)=e_{f}(3)$ \\
\hline 3,7 & $v_{f}(0)=v_{f}(1)=v_{f}(2)=v_{f}(3)+1$ & $e_{f}(0)=e_{f}(1)=e_{f}(2)=e_{f}(3)$ \\
\hline
\end{tabular}

Table 4 :

Illustration 2.8. The Flower graph $F L_{5}$ and its 4-cordial labeling is shown in Figure 4. 


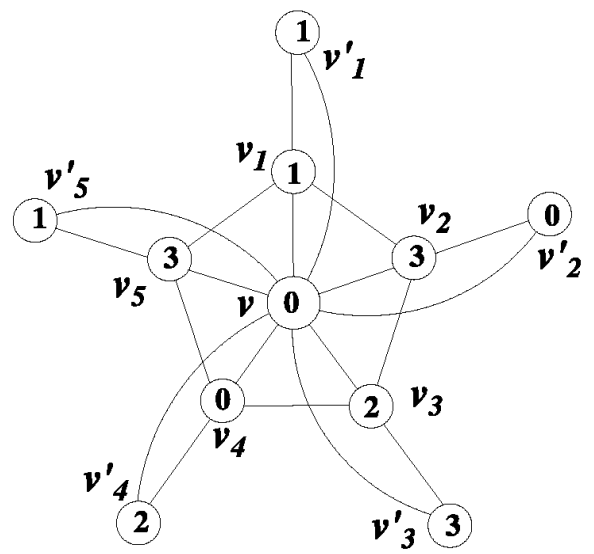

Figure 4: 4-cordial labeling of flower graph $\mathrm{FL}_{5}$.

\section{Concluding remarks}

To investigate similar results for other graph families and for other $k$-cordial labeling is an open area of research.

\section{REFERENCES}

1. J.A.Gallian, A dynamic survey of graph labeling, The Electronic Journal of Combinatorics, 19 (2016) \#DS6.

2. J.Gross and J.Yellen, Handbook of Graph Theory, CRC Press, (2004).

3. M.Hovey, A-cordial graphs, Discrete Mathematics, 93 (1991) 183-194.

4. K.K.Kanani and N.B.Rathod, Some new 4-cordial graphs, Journal of Mathematical and Computational Science, 4(5) (2014) 834-848.

5. N.B.Rathod and K.K.Kanani, Some path related 4-cordial graphs, International Journal of Mathematics and Soft Computing, 5(2) (2015) 21-27.

6. N.B.Rathod and K.K.Kanani, 4-cordial labelling of star, book and fan related graphs, Proceedings of 8th National Lavel Science Symposium, (2) (2015) 38-42.

7. N.B.Rathod and K.K.Kanani, 4-cordiality of some new path related graphs, International Journal of Mathematics Trends \& Technologies, 34(1) (2016) 5-8.

8. S.K.Vaidya and N.B.Vyas, Antimagic labeling of some path and cycle related graphs, Annals of Pure and Applied Mathematics, 3(2) (2013) 119-128.

9. S.K.Vaidya and P.L.Vihol, Radio labeling for some cycle related graphs, International Journal of Mathematics and Soft Computing, 2 (2) (2012) 11-24.

10. M.Z.Youssef, On k-cordial labeling, Australasian Journal of Combinatorics, 43 (2009) 31-37. 